\title{
Mini Review: Pharmacotherapy for Behavioral and Psychological Symptoms in Alzheimer' s Disease
}

Koji Hori ${ }^{1-3^{*}}$, Kimiko Konishi ${ }^{1,4}$, Hiroaki Tanaka ${ }^{2}$, Sachiko Yokoyama ${ }^{1}$, Mari Aoki ${ }^{1}$, Kazunari Azuma ${ }^{1}$, Koichi Zinbo ${ }^{1}$, Masaki Okada ${ }^{3}$ and Mitsugu Hachisu ${ }^{5}$

${ }^{1}$ Department of Psychiatry, Showa University Northern Yokohama Hospital, Kanagawa, Japan

${ }^{2}$ Department of Psychiatry, Showa University Fujigaoka Hospital, Kanagawa, Japan

${ }^{3}$ Department of Psychiatry, Hitachiumegaoka Hospital, Ibaraki, Japan

${ }^{4}$ Tokyo Metropolitan Tobu Medical Center for Persons with Developmental/Multiple Disabilities, Tokyo, Japan

${ }^{5}$ Department of Clinical Psychopharmacy, School of Pharmaceutical Sciences, Showa University, Tokyo, Japan

\begin{abstract}
Object: To show the clinical implications for pharmacotherapy for Behavioral and Psychological Symptoms of Dementia (BPSD) in Alzheimer's Disease (AD).

Background: In Japan, no medicine is permitted for BPSD in AD. On the contrary, atypical antipsychotics are inhibited because mortality rate increases when these medicines are prescribed for elderly demented patients

Methods and results: We reviewed our previous articles those reported the effects of aging and disease progress on BPSD and showed that effects of aging and disease progress caused the connections of the mood cluster (anxieties and affective disturbances) with the psychiatric cluster (delusions and hallucinations) and aggressiveness.
\end{abstract}

Conclusion: BPSD in AD was similar with bipolar features of mixed type or psychotic depression. We might prescribe medications for augmentation of depression to BPSD in AD.

Keywords: Behavioral and Psychological Symptoms of Dementia (BPSD); Alzheimer's Disease (AD); Pharmacotherapy; Augmentation

\section{Introduction}

In Japan, no medicine is permitted for behavioral and psychological symptoms of dementia (BPSD) in Alzheimer's disease (AD). On the contrary, atypical antipsychotics are inhibited because mortality rate increases when these medicines are prescribed for elderly demented patients [1]. However, antipsychotics are prescribed for BPSD such as delusions, hallucinations, agitations or aggressions with permission of proxies of $\mathrm{AD}$ patients. Therefore in this article, we showed and reviewed our previous articles those reported the effects of aging and disease progress on BPSD [2,3] and we recommended the clinical implication of the pharmacotherapy for BPSD in AD.

\section{Effects of Aging and Disease Progression on Behavioral Symptoms in Alzheimer's Disease}

In order to evaluate the effects of aging and disease progress on $\mathrm{BPSD}$ in $\mathrm{AD}$, eligible subjects consecutively referred $\mathrm{AD}[4]$ patients because of their BPSD were evaluate. We evaluated demographic data (sex difference, educational level, age at dementia onset, the age at the time of test and severity of dementia), cognitive function and BPSD at the study entry. The severity of dementia was evaluated using Functional Assessment Staging (FAST) [5]. Cognitive function was evaluated using the Mini-Mental State Examination (MMSE) [6], and BPSD were assessed by the Behavioral Pathology in Alzheimer's Disease Rating Scale (BEHAVE-AD) [7]. First, according to the age at the time of test, whole $\mathrm{AD}$ patients $(\mathrm{n}=79, \mathrm{WAD})$ group were divided into two groups, a relative older group in the WAD (with age the time of test were 81 and over, $n=40, O G$ ) and a relative younger group in WAD (with age the time of test were below $81, \mathrm{n}=39, \mathrm{YG}$ ) [2]. Then the factor analysis were conducted and showed that although in YG mood cluster (anxiety and phobia, affective disturbances), Psychotic cluster (delusions, hallucination), and behavioral cluster (inappropriate behaviors, aggressiveness, diurnal rhythm disturbance) were separated distinctively, in OG the psychotic cluster (delusions, hallucination) and anxiety and phobia constituted same factor. Moreover, aggressive behaviors and affective disturbances also constituted same factor in OG [2].

Secondary according to MMSE scores, WAD ( $\mathrm{n}=79)$ were divided into two groups, a relative higher performance group in the WAD (MMSE score of 12 or higher, $n=40, H P G$ ) and a relative lower performance group in the WAD (MMSE cores was below 12, $n=39$, LPG) $[2,3]$. We conducted the same procedures again. Although in HPG mood cluster (anxiety and phobia, affective disturbances), Psychotic cluster (delusions, hallucination), and behavioral cluster (inappropriate behaviors, aggressiveness, diurnal rhythm disturbance) were separated distinctively, in LPG the psychotic cluster (delusions, hallucination), and mood cluster (anxiety and phobia, affective disturbance) and aggressiveness constituted same factor [3].

\section{Discussion}

From these results, we considered that the mood cluster becomes connected to the psychiatric cluster and aggressiveness according

*Corresponding author: Koji Hori, Department of Psychiatry, Showa University Northern Yokohama Hospital 35-1 Chigasakichuo, Tsuzukiku, Yokohama-City, Kanagawa, 224-8503, Japan, Tel: +81-45-949-7000; Fax: +81-45-949-7927, E-mail: kojihori@med.showa-u.ac.jp

Received June 12, 2013; Accepted September 06, 2013; Published September 09, 2013

Citation: Hori K, Konishi K, Tanaka H, Yokoyama S, Aoki M, et al. (2013) Min Review: Pharmacotherapy for Behavioral and Psychological Symptoms in Alzheimer's Disease. Brain Disord Ther 2:106. doi:10.4172/2168-975X.1000106

Copyright: (C) 2013 Hori K, et al. This is an open-access article distributed under the terms of the Creative Commons Attribution License, which permits unrestricted use, distribution, and reproduction in any medium, provided the original author and source are credited. 
to that the patients with $\mathrm{AD}$ become older and $\mathrm{AD}$ progresses. Generally speaking, depressive syndrome (anxieties, phobias, and affective disturbances) are thought to be diminished according to aging and the progression of dementia [8]. However, we consider that depressive symptoms are not disappeared but connected with psychotic symptoms, i.e., depressive symptoms turn up as psychotic symptoms. We refer this phenomenon "implicit depression and explicit psychosis" [3]. Moreover, this feature is also related with mixed states of depression and mania because manic symptoms (aggressiveness, delusion, hallucination) were connected with depressive symptoms (anxiety, affective disturbance) $[9,10]$. In fact $\mathrm{Ng}$ et al. reported that there were several commonalities in pathophysiological processes of bipolar disorders and dementia [9]. In fact Dorey et al. reported that some BPSD could be the consequence of both dementia and an undiagnosed comorbid bipolar disorder, or pre-existing bipolar diathesis pathoplastically altering the clinical expression of dementia [10].

We will place the suggestion on treatment of $\mathrm{AD}$ from our clinical implications in our two reports. In case of patients who show psychotic symptoms at relative severe stage of $\mathrm{AD}$ or with relative older age, it is better to prescribe for depression but not for psychosis. Moreover, aging and the progression of $\mathrm{AD}$ are irreversible processes. Therefore, the connections of mood cluster and psychotic cluster might be better generalized. We might prescribe "augmentation" medications for BPSD. In Japan some anticonvulsants (sodium valproate, lamotrigine), some antipsychotic agents (olanzapine, aripiprazole) and lithium carbonate are allowed for treatment of bipolar spectrum, i.e., augmentation for depression. Therefore, because depressive state is related not with monopolar depression but with bipolar spectrum or bipolar depression, we should prescribe not the antidepressants but augumentations. Therefore, if so, not antidepressant but atypical antipsychotics should be prescribed for augmentation for depressive patients. Moreover, atypical antipsychotics should be prescribed as for augmentation for depression $[11,12]$. Of course, these medications must have no or little anticholinergic activity; those induce cognitive dysfunctions [13] and BPSD [14] in AD patients. We refer this that antipsychotic should be prescribe not for psychosis but for depression.

However, FDA issued a warning about prescription of antipsychotic agents to demented elderly patients, because of increasing the mortality of the patients [1] therefore, we should not prescribe antipsychotic agents at first. Therefore we should prescribe antidepressants, Yokukansan (Japanese herbal medicine) and anticonvulsants or antipsychotic agents for augmentation for depression. However, if there were no effects with antidepressants, Yokukansan and anticonvulsants, we might prescribe antipsychotics as augmentation for depression. In fact, there are many reports that antidepressants ameliorate cognitive function in mice [15] as well as BPSD in human [16-19]. For example, Burke et al. reported selective serotonin reuptake inhibitor was effective to reduce psychotic symptoms in depression and psychosis complicated with dementia those were not responded to neuroleptics [15]. The effect of Yokukansan is also reported to ameliorate BPSD such as delusion and hallucination [20,21]. Perhaps, serotonergic modulation might bring the amelioration of BPSD in $\mathrm{AD}[22]$.

There are several limitations in the present study these two reports were without control subjects, not observing longitudinal course of cognitive dysfunctions but only cross-sectional. Moreover, these two studies didn't include "bipolarity" as predictive values. Further investigations should be required to delineate relationship between bipolarity and the features of BPSD in AD in order to better medications for BPSD in AD. We are now conducting observations in a larger patient cohort to evaluate the relationship between bipolarity and the features of BPSD in AD.

\section{Conflicts of Interest}

Koji Hori received lecture fees from Eisai Co., Ltd., Pfizer Japan Inc., Novartis Pharma K.K., Daiichi Sankyo Inc., Ono Pharmacuetical Co., Ltd., Janssen Pharmaceutical K.K., Yoshitomi Yakuhin Co., and Mitsubishi Tanabe Pharma Co. Mitsugu Hachisu received funding from Astellas Pharma Inc., Meiji Seika Pharma Co., Ltd., Dainippon Sumitomo Pharm Co., Ltd., Eli Lilly Japan K.K., and Shionogi \& Co., Ltd., and received lecture fees from Meiji Seika Pharma Co., Ltd. and Mitsubishi Tanabe Pharma Co.

\section{Acknowledgment}

Funding for this study was received from Eisai Co., Ltd., Daiichi Sankyo Inc. and Ono Pharmacuetical Co., Ltd.

\section{References}

1. Schineider LS, Dagerman K, Insel P (2005) Risk of death with atypical antipsychotics drug treatment for dementia: meta-analysis of randomaized placebo-controlled trials. JAMA 294: 1934-1943.

2. Konishi K, Hori K, Oda T, Tominaga I, Asaoka T, et al. (2009) Effects of aging on behavioral symptoms in Alzheimer's disease. Psychogeriatrics 9: 11-16.

3. Hori K, Konishi K, Tomioka H, Tani M, Minegishi G, et al. (2012) Mood symptoms are related to psychotic symptoms in severe Alzheimer's disease. J Addict Res Ther S5: 002

4. McKhann G, Drachman D, Folstein M, Katzman R, Price D, et al. (1984) Clinica diagnosis of Alzheimer's disease: Report of the NINCDS-ADRDA Work Group under the auspices of Department of Health and Human Services Task Force in Alzheimer's disease. Neurology 56: 939-944.

5. Reisberg B (1998) Functional assessment staging (FAST). Psychopharmaco Bull 24: 189-198.

6. Folstein MF, Folstein SE, McHugh RP (1975) "Mini-Mental State." A practical method for grading the cognitive state of patients for the clinician. J Psychiatry Res12: 95-112.

7. Reisberg B, Borenstein J, Salob SP, Ferris SH, Franssen E, et al. (1987) Behavioral symptoms in Alzheimer's disease: phenomenology and treatment J Clin Psychiatry 48: 9-15.

8. Teri L, Larson EB, Reifler BV (1988) Behavioral disturbance in dementia of the Alzheimer's type. J Am Geriatr Soc 36: 1-6.

9. Ng B, Camacho A, Lara DR, Brunstein MG, Pinto OC, et al. (2008) A case series on the hypothesized connection between dementia and bipolar spectrum disorders: bipolar type VI ? J Affect Disord 107: 307-315.

10. Dorey JM, Beauchet OB, Anterion CT, Rouch I, Krolak-Saimon P, et al. (2008) Behavioral and psychological symptoms of dementia and bipolar spectrum disorders: review of the evidence of a relationship and treatment implication CNS Spectr 13: 796-803.

11. Papakostas GI, Shelton RC, Smith J, Fava M (2007) Augmentation of antidepressants with atypical antipsychotic medications for treatment-resistan major depressive disorder: a meta-analysis. J Clin Psychiatry 68: 826-831.

12. Shelton RC, Papakostas GI (2008) Augmentation of antidepressants with atypical antipsychotic medications for treatment-resistant major depressive disorder. Acta Psychiatr Scand 117: 253-259.

13. Konishi K, Hori K, Uchida H, Watanabe K, Tominaga I, et al. (2010) Adverse effects of anticholinergic activity on cognitive functions in Alzheimer's disease. Psychogeriatrics 10: 34-38 
Citation: Hori K, Konishi K, Tanaka H, Yokoyama S, Aoki M, et al. (2013) Mini Review: Pharmacotherapy for Behavioral and Psychological Symptoms in Alzheimer's Disease. Brain Disord Ther 2:106. doi:10.4172/2168-975X.1000106

Page 3 of 3

14. Hori K, Konishi K, Watanabe K, Uchida H, Tsuboi T, et al. (2011) Influence of anti-cholinergic activity in serum on clinical symptoms of Alzheimer's disease. Neuropsychobiology 63: 147-153.

15. Flood JF, Checrkin A (1987) Fluoxetine enhances memory processing in mice. Psychopharmacology 93: 36-43.

16. Burke W, Dewan V, Wengel SP, Rocaforte WH, Nudolny GC, et al. (1997) The use of selective serotonin reuptake inhibitor for depression and psychosis complicated dementia. Int J Geriatr Psychiatry 12: 519-525.

17. Trappler B, Vinuela LM (1997) Fluvoxamine for stereotypic behaviora in patients with dementia. Ann Pharmacother 31: 578-581.

18. Ramadam FH, Naughton BJ, Bassanelli AG (2000) Treatment of verbal agitation with a selective serotonin reuptake inhibitor. J Geriatr Psychiatry Neurol 13: 56-59.
19. Ramasubbu $R$ (2004) Cerebrovascular effects of selective serotonin reuptake inhibitors: a systemic review. J Clin Psychiatry 65: 1642-1653.

20. Mizukami K, Asada T, Kinoshita T, Tanaka K, Sonohara K, et al. (2009) A randomized cross-over study of a traditional Japanese medicine (kampo), yokukansan, in the treatment of behavioral and psychological symptoms of dementia. Int J Neuropsychopharmacology 12: 191-199.

21. Okahara K, Ishida $Y$, Hayashi $Y$, Inoue T, Tsuruta K, et al. (2010) Effects of yokukansan on behavioral and psychological symptoms of dementia in regular treatment for Alzheimer's disease. Prog Neuropsychopharmacol Biol Psychiatry 34: 532-536.

22. Ueki A, Ueno H, Sato N, Shinjo H, Morita $Y$ (2007) Serotonin transporter gene polymorphism and BPSD in mild Alzheimer's disease. J Alzheimers Dis 12: $245-253$ 